\title{
Distribution of Gingival Inflammation in Mouth Breathing Patients: An Observational Pilot Study
}

\author{
Rajinder Kumar Sharma, Anu Bhatia, Shikha Tewari, Satish Chandar Narula \\ Pandit Bhagwat Dayal Sharma University of Health Sciences, Rohtak, Haryana 124000, India \\ Correspondence e-mail to: bhatiaanu01@gmail.com
}

\begin{abstract}
Mouth breathing has been reported to affect gingival health in children. However, studies on the effect of mouth breathing in adult patients are scarce. Objective: To examine the relationship between mouth breathing and gingival condition and to evaluate the distribution of gingival inflammation in young adult mouth breathing patients. Methods: Study groups comprised of participants with mouth breathing (test group) and nose breathing (control group) patients with gingivitis. Both the groups underwent periodontal examination. PI, GI and BOP \% sites were recorded and analyzed statistically for the differences in mean values. Results: Mouth breathing patients showed higher full mouth GI and BOP scores. Upper anterior segment in mouth breathing patients showed highest GI and BOP followed by lower anterior segment, lower posterior and upper posterior region. Conclusion: Within the limits of present study, our findings suggest that relative to control group participants, test group i.e. patients with mouth breathing had higher gingival inflammation and bleeding sites in upper anterior region.
\end{abstract}

Keywords: gingivitis, mouth breathing, young adult

\section{INTRODUCTION}

Mouth breathing may be regarded as benign, harmless, self-effacing and unsuspecting way of breathing. Mouth breathing syndrome is a term used when mouth supplements the nose for breathing. ${ }^{1}$ Oral breathing patterns exclusively are rare or non-existent. ${ }^{1}$ For many, it hardly matters whether breathing is done through mouth or nose. Consequently, the debilitating effects of mouth breathing may be disregarded and thus, are failed to be noticed even by dental professionals.

Primary etiological factor for chronic gingivitis is dental plaque; however, anything that favors plaque formation, accumulation and retention will perpetually aggravate existing gingivitis. Results of epidemiological studies indicate that mouth breathing may cause an increase in susceptibility of gingival inflammation. ${ }^{2,3}$ Although the definite mechanism of the damaging effects of mouth breathing on gingiva is still unknown; irritation from surface dehydration, reduced resistance of epithelium to plaque, and the absence of cleansing effects of saliva have been suggested as some of the reasons for increased gingivitis prevalence in mouth breathers. ${ }^{3,4}$ Evidence regarding the relationship between mouthbreathing and gingivitis has contradictory positions. While Sutcliffe et al reported a total lack of correlation between mouth breathing and gingivitis, others like Alexander et al proposed a partial association asserting that mouth breathing in itself is of no consequence except in the presence of crowding and calculus. ${ }^{5,6}$ However, previous studies observed a definite association between mouth breathing and chronic gingivitis. . $^{2,7}$

In light of the paucity of definite research information concerning the effect of mouth breathing on gingival inflammation, the current pilot study was conducted with an aim to find out the distribution of gingival inflammation within the oral cavity in mouth breathers and to explore any association between mouth breathing, plaque level and gingivitis. 


\section{METHODS}

\section{Study design and sample}

The study population consisted of patients who attended the outpatient section of the Department of Periodontics and Oral Implantology, Post Graduate Institute of Dental Sciences, Rohtak, India. Study was conducted in agreement with the ethical principles embodied in Helsinki Declaration of 1975, as revised in 2008, and was approved by the institutional review board (PGIDS/2013/IEC/94, 2013). The study was conducted between April 2013 and August 2014. The study consisted of two groups: test group comprising of 30 mouth breathing patients with incompetent lip seal, with an age range of 20-35 years and age matched control group of 34 nose breathing participants.

\section{Diagnosis of mouth breathing}

Diagnosis of mouth breathing was made on the basis of history and clinical examination of the patients. Patients were enquired if in their opinion, they were mouth breathers or not, whether they sleep with their mouth open and also whether on waking they had dryness of mouth. Clinical examination included several diagnostic tests for mouth breathing. Firstly, the subjects were asked to breathe while closing one of the nostrils with lips sealed. Nose breathers demonstrated good control of alar muscle which was absent in mouth breathers. Secondly, mirror test. Double sided mirror was held horizontally below the nostrils of patients who were instructed to breathe normally. Fogging on lower side of mirror suggested mouth breathing. ${ }^{8}$ Thirdly, Butterfly test. Butterfly shaped piece of cotton was placed below the nostrils on upper lip. Fluttering of cotton wisp indicated breathing pattern. If the upper fibres were displaced then the breathing was considered through the nose and if lower fibres quivered, it suggested mouth breathing. ${ }^{9}$ Forthly, Water holding test. Participants were asked to fill the mouth with water and hold it for three to five minutes. Mouth breathers suffered difficulty completing this task whereas nasal breathers did it with relative ease. ${ }^{8}$

For fulfilling the criteria of mouth breathing, subjects were required to give a positive history along with minimum of two clinical tests suggestive of mouth breathing habit.The inclusion criteria for the study were presence of $>20$ teeth; systemically healthy patients with gingivitis in the age group of 20-35 years; and no periodontal treatment within past 6 months prior to inclusion into the study. The exclusion criteria included presence of clinical signs or symptoms of any acute infection in the oral cavity; use of systemic antibiotics or anti-inflammatory therapy in the last 3 months before start of study; any known systemic (e.g. hepatic, renal, haematological or cardiovascular) disease; pregnancy and lactation; systemic conditions with gingival manifestations and non-plaque induced gingival inflammation; xerostomia and drugs reported to cause it; and current or former smokers.

\section{Periodontal measurements}

The full-mouth periodontal examination of all individuals was done by measurement of Loe and Silness gingival index (GI) and Silness and Loe plaque index (PI) on all teeth except third molars. These indices were recorded on four sites (mesiobuccal, midbuccal, distobuccal and palatal aspects) around each tooth with University of North Carolina-15 (UNC-15) periodontal probe. Bleeding sites were registered on six sites per tooth in a dichotomous way, and scores were expressed as the percentage of positive sites per patient (BOP \%).

In order to ensure investigator blinding as well as to preclude inter examiner variability, oral examination was carried out by one investigator (AB). Investigator was masked to the study group to which the patient belongs. Examiner reproducibility was determined by carrying out double clinical periodontal data recording on ten patients. Operator calibration for GI was based on $>85 \%$ intra-examiner exact reproducibility.

\section{Statistical analysis}

Post hoc power analysis was done using statistical software (G power 3.1.9.2). With a sample size of 64 and significance level of two-sided $\alpha=0.05$, fixedeffect size was calculated taking upper anterior GI as primary outcome variable. With these measurements, statistical power exceeded $95 \%$, with allocation ratio of $1: 1$ between two groups. Results are reported as mean \pm standard deviation. The normality of distribution of data was examined using Shapiro-Wilk test. Data was found to be non-normally distributed. All the measurements were subjected to intergroup comparisons and analyzed by Mann-Whitney U test. All statistical analyses were carried out using statistical software (SPSS V. 19) with a two-tailed p value of 0.05 used as a threshold for significance.

\section{RESULTS}

Study group comprised 64 adult patients. Table 1 illustrates the demographic and clinical parameters among the mouth breathing and nose breathing groups. Average age of patients in test group was 24.6 years and average age of control group was 25.0 years. Full mouth plaque score between two groups did not differ significantly. Patients with mouth breathing demonstrated significantly higher full mouth GI and BOP $(\%)(p<0.05)$ than nose breathing group. The dentition was analyzed after being divided into four segments - two anterior segments - each comprising of either upper or lower anterior teeth and two lateral zones- upper and lower, comprising of remaining teeth.

Table 2 showed comparison of segment wise periodontal parameters between two groups. Control group showed higher plaque scores as well as gingival index in lower posterior area followed by lower anterior regions. 
Table 1. Comparison of demographic and clinical periodontal parameters among nose breathing and mouth breathing group.

\begin{tabular}{lcc}
\hline Parameters & \multicolumn{2}{c}{ Group } \\
\cline { 2 - 3 } & $\begin{array}{c}\text { Nose breathing } \\
(\mathrm{n}=34) \\
\text { Mean } \pm \text { SD }\end{array}$ & $\begin{array}{c}\text { Mouth breathing } \\
(\mathbf{n}=\mathbf{3 0}) \\
\text { Mean } \pm \text { SD }\end{array}$ \\
\hline Age (years) & $25.02 \pm 3.78$ & $24.67 \pm 3.51$ \\
Male : Female & $1.31: 1$ & $1.66: 1$ \\
Full Mouth PI & $1.47 \pm 0.50$ & $1.48 \pm 0.62$ \\
Full Mouth GI & $1.71 \pm 0.37$ & $1.93 \pm 0.31^{*}$ \\
Fu11 Mouth & $74.10 \pm 20.20$ & $83.50 \pm 18.91^{*}$ \\
BOP\% & & \\
$* p<0.05$ & &
\end{tabular}

Table 2. Comparison of segment wise periodontal parameters among nose breathing and mouth breathing group

\begin{tabular}{llrr}
\hline Segment & Parameters & \multicolumn{2}{c}{ Group } \\
\cline { 3 - 4 } & & $\begin{array}{c}\text { Nose } \\
\text { breathing } \\
\text { Mean } \pm \text { SD }\end{array}$ & $\begin{array}{c}\text { Mouth } \\
\text { breathing } \\
\text { Mean } \pm \text { SD }\end{array}$ \\
\hline Upper anterior & PI & $1.34 \pm 0.48$ & $1.52 \pm 0.62$ \\
& GI & $1.58 \pm 0.37$ & $2.09 \pm 0.28^{*}$ \\
& BOP (\%) & $67.21 \pm 24.13$ & $91.88 \pm 8.01^{*}$ \\
Upper posterior & PI & $1.44 \pm 0.56$ & $1.33 \pm 0.61$ \\
& GI & $1.65 \pm 0.45$ & $1.68 \pm 0.35$ \\
& BOP (\%) & $73.93 \pm 22.84$ & $77.75 \pm 24.85$ \\
Lower anterior & PI & $1.52 \pm 0.58$ & $1.54 \pm 0.70$ \\
& GI & $1.77 \pm 0.44$ & $1.99 \pm 0.46^{*}$ \\
& BOP $(\%)$ & $77.09 \pm 22.46$ & $85.60 \pm 21.57^{*}$ \\
Lower posterior & PI & $1.54 \pm 0.53$ & $1.40 \pm 0.61$ \\
& GI & $1.80 \pm 0.47$ & $1.70 \pm 0.40$ \\
& BOP $(\%)$ & $76.30 \pm 21.91$ & $78.20 \pm 24.98$ \\
\hline
\end{tabular}

Upper posterior segment still showed higher values of PI and GI than upper anterior region in control group. In test group, highest plaque score was found in lower anterior region followed by upper anterior, lower posterior and upper posterior segments. GI value, however, was highest in upper anterior region as was BOP $\%$ sites in test group. Also, lower anterior segment showed GI higher score as compared to lower posterior area. Upper posterior region in mouth breathing group exhibited lowest score of GI and $\mathrm{BOP} \%$ sites.

In mouth breathing patients, facial surfaces of upper anterior teeth showed greater PI, GI and BOP $\%$ as compared to palatal surfaces (Table 3). Also, interdental sites of facial surfaces had higher scores than mid-facial regions in test group.
Table 3. Comparison of periodontal parameter in upper anterior region in mouth breathing group

\begin{tabular}{llc}
\hline Parameter & $\begin{array}{l}\text { Facial surface } \\
\text { Mean } \pm \text { SD }\end{array}$ & $\begin{array}{l}\text { Palatal surface } \\
\text { Mean } \pm \text { SD }\end{array}$ \\
\hline PI & $1.57 \pm 0.65$ & $1.46 \pm 0.59$ \\
GI & $2.17 \pm 0.27$ & $2.00 \pm 0.33^{*}$ \\
BOP $(\%)$ & $95.11 \pm 9.80$ & $88.10 \pm 16.14^{*}$ \\
\hline
\end{tabular}

\section{DISCUSSION}

Although a correlation between mouth breathing and oral conditions in children has been reported, there are few such studies in young adults. Also, there are only a few reports dealing with the regional distribution of gingival inflammation in the oral cavity. The aim of this study was thus to examine the relationship between mouth breathing and gingival condition in young patients and to assess the distribution pattern of inflammation in such patients.

Mouth breathing habit is widely reported among school going children. ${ }^{10}$ If left untreated, it can lead to morphological alterations in the facial growth and various adverse effects on physiological, social and mental health. ${ }^{11}$ Compared with the previous studies where mouth breathing patients were recruited from younger age groups (less than 14 years), participants in our study were older and thus, findings of this study may be applicable to a broader range of population with similar characteristics. The age and gender distribution was reasonably well balanced in both the groups. There were more females in mouth breathing group. Study population belonged to same ethnic background and was recruited from middle class families.

Results of our study showed higher plaque score in lower dentition as compared to upper dentition in control group. Lower posterior areas showed greatest accumulation of plaque followed by lower anterior region. Upper posterior region harbored more plaque compared to upper anterior area. In nose breathing patients, pattern of distribution of gingival inflammation and BOP \% sites closely followed the areas of plaque accumulation. Thus, upper anterior area showed least gingival inflammation and bleeding sites in nose breathing patients. These observations were similar to the results of previous studies that demonstrated higher levels of plaque on molar, lingual and posterior surfaces than on anterior teeth. ${ }^{12,13}$

Contrary to above observation, in mouth breathing group, upper anterior area showed greatest GI score and $\mathrm{BOP} \%$ sites. This observation of our study is consistent with results of previous studies that demonstrated maxillary anterior area as most susceptible to gingival inflammation in mouth breathing subjects. ${ }^{2,7,14}$ In lower arch, lower anterior segment showed greater 

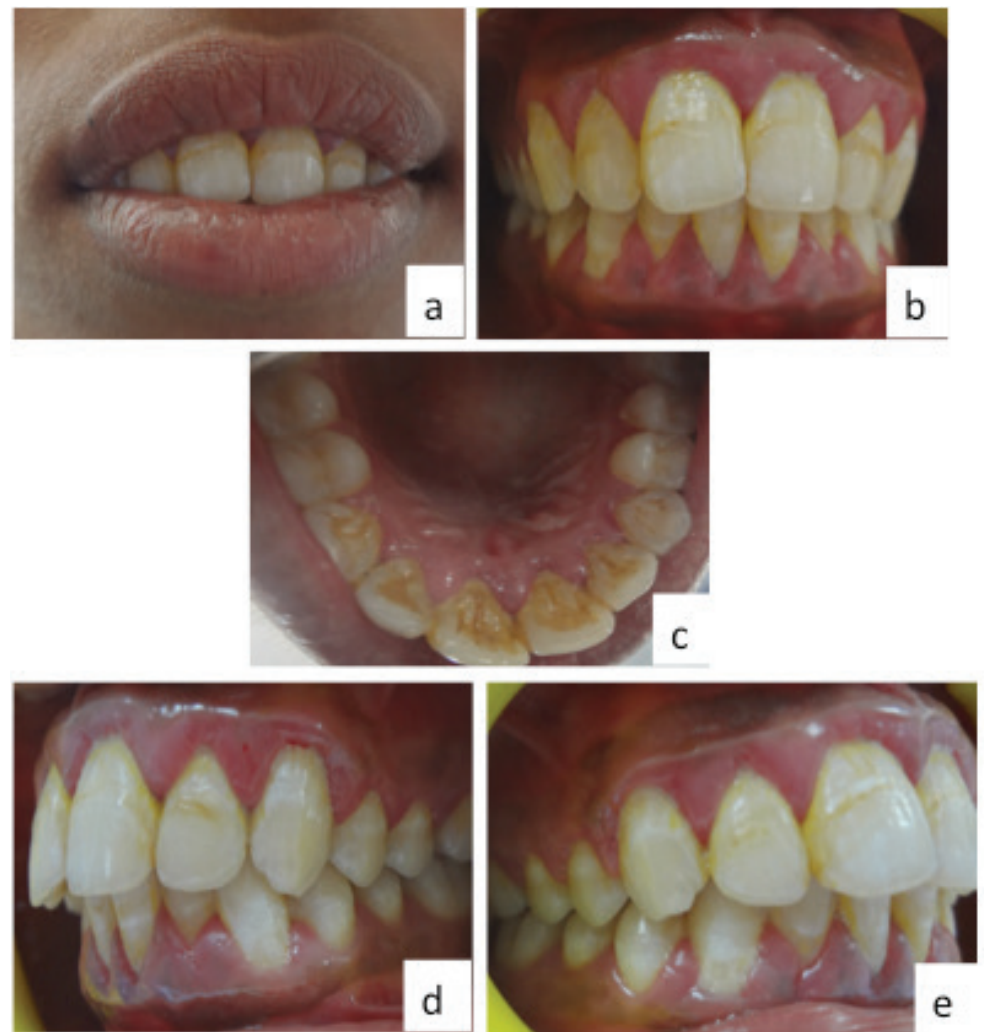

Figure 1. Mouth breathing patient a) Lip position at rest; b) Anterior region showing severe gingival inflammation; c) Maxillary palatal aspect; d) Left lateral region showing inflammation upto premolar; e) Right lateral region showing inflammation upto premolar

gingival inflammation and bleeding as compared to corresponding posterior segment. Noticeably, the result of our study also showed that the upper posterior area in mouth breathing patients had least amount of gingival inflammation. Plaque scores in this segment was also least as compared to rest of the areas.

The importance of normal hydration state of periodontium in the maintenance of periodontal health is underscored by the increase in gingival inflammation that inevitably follows the chronic mouth breathing habit. Also, in a recent study mizutani et al has observed that xerostomia was related to gingival disease activity and \% BOP through the accumulation of dental plaque. ${ }^{15}$ The great propensity for gingival inflammation in mouth breathers probably stems from dryness of affected area causing the loss of the protective powers of saliva. Saliva has an essential role in protecting the tissues against dessication. Alteration of homeostatis of hydration state of gingiva can be caused by continuous exposure to the dry air of tissues of oral cavity. Without the shielding effects of adequate salivary flow, periodontal tissues might become prone to disease. Patients with chronic mouth breathing habit might suffer from dry mouth which could eventually create a predisposition to oral infection and progressive gingivitis. ${ }^{15}$ Similar observation was made in Al-awadi et al's study who noticed increase in PI and GI in mouth breathing group as compared to nose breathing group and attributed this to reduced salivary flow rate in mouth breathers. ${ }^{16}$ Since gingivitis is a disease of microbial origin, the mouth breathing related (increase in) gingival inflammation could be an expression of increased or altered microbial population dynamics in the oral cavity.

Previous studies by Wagaiyu et al and Gulati et $\mathrm{al}^{7}$ have assessed the effects of mouth breathing, lip competency and upper lip coverage of maxillary incisor teeth simultaneously on gingival health. ${ }^{2-7}$ Both these studies revealed that mouth breathing patients with incompetent lip seal and wide exposure of maxillary labial gingiva had more gingival inflammation. Our study population composed of test group including mouth breathers with incompetent lip seal and gingival exposure in order to create homogeneity of test group sampled and also to lessen the influence of other confounding factors.

Though the distribution of gingival units bearing inflammation as well as severity of inflammation in these units was uniform to a large extent in mouth breathing patients, there was no characteristic clinical picture depicting inflammatory changes in the marginal tissues of this population. Clinical picture in mouth breathing patients demonstrated that marked gingival inflammation was confined to anterior 
regions of maxilla. Interdental papilla and marginal gingiva belonging to areas exposed to the drying effects of inspired air also exhibited marked gingival inflammation in mouth breathing patients. Incidence and severity of ginigival inflammation was significantly lower in the areas not supposed to be in the pathway of inspired air during mouth breathing.

When upper anterior area is investigated in mouth breathers, facial surface showed higher GI score and $\mathrm{BOP} \%$ sites than the palatal surface. On further analyses of the facial surface, inter-dental sites had higher score than mid-facial sites in mouth breathers. Thus, upper anterior area in mouth breathing patients demands attention and can be regarded as a seat for early detection or screening of gingival inflammation. Dental plaque formation and gingival inflammation are the earliest and most common indicators of periodontal disease in children and adolescents. Not only are individuals with mouth breathing susceptible to gingival inflammation, but also such changes start manifesting at quite young age in life. Therefore, controlling oral health behaviour and evaluating mouth breathing would effectively prevent periodontal disease at an early stage in young people. The stringent inclusion and exclusion criteria, study population derived from the same ethnic background, and exclusion of smokers were some of the strengths of present study. The results of this study should be interpreted cautiously in light of its limitations. As this study was cross-sectional, it remains uncertain as to whether mouth breathing is the cause of gingival inflammation. Prospective cohort studies may be required to fully elucidate the mechanism involved.

\section{CONCLUSION}

In conclusion, the results of present study indicate that the mouth breathing patients with incompetent lip seal had higher scores of gingival inflammation in adults, especially in upper anterior segment. Also, the distribution of gingival inflammation in such patients differs when compared to nasal breathers. Thus, clinician should be more vigilant while screening such individuals as mouth breathing could be one of the risk factors in gingivitis.

\section{CONFLICT OF INTEREST}

There are no potential conflicts of interest or any financial or personal relationships with other people or organizations that could inappropriately bias the conduct and findings of this study.

\section{REFERENCES}

1. Abreu RR, Rocha RL, Lamounier JA, Guerra AF. Etiology, clinical manifestations and concurrent findings in mouth-breathing children. J Pediatr. (Rio J) 2008;84:529-35.

2. Wagaiyu EG, Ashley FP. Mouth-breathing, lip seal and upper lip coverage and their relationship with gingival inflammation in 11-14year-olds. J Clin Periodontol. 1991;18:698-702.

3. Jacobson L. Mouthbreathing and gingivitis. 1. Gingival conditions in children with epipharyngeal adenoids. J Periodontol Res.1973;8:269-77.

4. Haytec MC, Oz IA. Atypical streptococcal infection of gingiva associated with chronic mouth breathing. Quintessence Int. 2007;38: E577-82.

5. Sutcliffe P. Chronic anterior gingivitis. An epidemiological study in schoolchildren. Br Dent J. 1968;125:47-55.

6. Alexander AG. Habitual mouth breathing and its effect on gingival health. Paradontologie. 1970;24:49-55

7. Gulati MS, Grewal N, Kaur A. A comparative study of effects of mouth breathing and normal breathing on gingival health in children. J Indian Soc Pedod Prev Dent. 1998;16:72-83.

8. Bianchini AP, Guedes ZCF, Vieira MM. A study on the relationship between mouth breathing and facial morphological pattern. Rev Bras Otorrinolaringol. 2007:73;500-5.

9. Bhatia A, Sharma R K, Tewari S, Narula SC. A randomized clinical trial of salivary substitute as an adjunct to scaling and root planing for management of periodontal inflammation in mouth breathing patients. J Oral Sci. 2015;57:241-7.

10. Garde JB, Suryavanshi RK, Jawale BA, Deshmukh V, Dadhe DP, Suryavanshi MK. An epidemiological study to know the prevalence of deleterious oral habits among 6 to 12 year old children. J Int Oral Health. 2014;6:39-43.

11. Jefferson Y. Mouth breathing: adverse effects on facial growth, health, academics, and behavior. Gen Dent. 2010;58:18-25.

12. Prasad KV, Sreenivasan PK, Patil S, Chhabra KG, Javali SB, DeVizio W. Removal of dental plaque from different regions of the mouth after a 1- minute episode of mechanical oral hygiene. Am J Dent. 2011;24:60-4.

13. Claydon NC. Current concepts in toothbrushing and interdental cleaning. Periodontol 2000. 2008; 48:10-22.

14. Solomon O N, Patricia OA. Uncommon causes of gingivitis. J Dent Oral Hyg. 2011;3:65-8.

15. Mizutani S, Ekuni D, Tomofuji T et al. Relationship between xerostomia and gingival condition in young adults. J Periodontal Res. 2015;50:74-9.

16. Al-Awadi RN, Al-Casey M. Oral health status, salivary physical properties and salivary Mutans Streptococci among a group of mouth breathing patients in comparison to nose breathing. J Bagh College Dent. 2013;25:152-9.

(Received February 2, 2016; Accepted June 24, 2016) 